\title{
Effect of Pitavastatin on Preventing Ischemic Complications with Carotid Artery Stenting: A Multicenter Prospective Study-EPOCH-CAS Study
}

\author{
Katsutoshi Takayama - Waro Taki • Naoki Toma - Ichiro Nakahara • \\ Masayuki Maeda - Hiroshi Tanemura - Terumasa Kuroiwa · Keisuke Imai • \\ Masahiko Sakamoto - Ichiro Nakagawa - Osamu Masuo - Kaoru Myouchin • \\ Takeshi Wada $\cdot$ Hidenori Suzuki
}

Received: 13 October 2013/Accepted: 14 November 2013/Published online: 10 December 2013

(C) Springer Science+Business Media New York and the Cardiovascular and Interventional Radiological Society of Europe (CIRSE) 2013

\begin{abstract}
Purpose Periprocedural ischemic stroke is one problem associated with carotid artery stenting (CAS). This study was designed to assess whether preoperative statin therapy reduces the risk of periprocedural ischemic complications with CAS.

Methods In this prospective study at 11 centers, patients with carotid artery stenosis (symptomatic $\geq 50 \%$, asymptomatic $\geq 80 \%$ ) and a high risk of carotid endarterectomy but without previous statin treatments were divided into two groups by low-density lipoprotein cholesterol (LDL-C)
\end{abstract}

This study was conducted on behalf of the EPOCH-CAS Investigators. The members of EPOCH-CAS Investigators are given in Appendix.

Trial Registration: UMIN Clinical Trials Registry: UMIN000004113. https://upload.umin.ac.jp/cgi-open-bin/ctr/ctr.cgi?function= brows \&action=brows $\&$ recptno $=$ R000004946\&type $=$ summary \&language $=\mathrm{E}$.

K. Takayama $(\bowtie) \cdot$ K. Myouchin

Department of Radiology and Interventional Neuroradiology,

Ishinkai Yao General Hospital, 1-41 Numa Yao,

Osaka 581-0036, Japan

e-mail: takayamaneuroivs@par.odn.ne.jp

W. Taki $\cdot$ N. Toma $\cdot$ H. Tanemura $\cdot$ H. Suzuki

Department of Neurosurgery,

Mie University Graduate School of Medicine,

Tsu, Japan

I. Nakahara

Department of Neurosurgery, Kokura Memorial Hospital,

Fukuoka, Japan

M. Maeda

Department of Radiology, Mie University Graduate School of

Medicine, Tsu, Japan levels. With LDL-C $\geq 120 \mathrm{mg} / \mathrm{dl}$, the pitavastatin-treated (PS) group received pitavastatin at $4 \mathrm{mg} /$ day. With LDL-C $<120 \mathrm{mg} / \mathrm{dl}$, the non-PS group received no statin therapy. After 4 weeks, both groups underwent CAS. Frequencies of new ipsilateral ischemic lesions on diffusion-weighted imaging within $72 \mathrm{~h}$ after CAS and cerebrovascular events (transient ischemic attack, stroke, or death) within 30 days were assessed.

Results Among the 80 patients enrolled, 61 patients (PS group, $n=31$; non-PS group, $n=30$ ) fulfilled the inclusion criteria. New ipsilateral ischemic lesions were identified in 8 of 31 patients $(25.8 \%)$ in the PS group and 16 of 30 patients $(53.3 \%)$ in the non-PS group $(P=0.028)$. Cerebrovascular events occurred in 0 patients in the PS group and in 3 of 30 patients $(10.0 \%)$ in the non-PS group $(P=0.071)$. Multivariate analyses demonstrated the pitavastatin treatment $(\beta=0.74,95 \%$ confidence interval $0.070-1.48, P=0.042$ ) to be an independent factor for decreasing post-CAS ischemic lesions.

\author{
T. Kuroiwa \\ Department of Neurosurgery, Osaka Medical College, Takatsuki, \\ Japan \\ K. Imai \\ Department of Neurology, Kyoto First Red Cross Hospital, \\ Kyoto, Japan \\ M. Sakamoto · T. Wada \\ Department of Radiology, Nara Medical University, Kashihara, Japan \\ I. Nakagawa \\ Department of Neurosurgery, Nara Medical University, \\ Kashihara, Japan \\ O. Masuo \\ Department of Neurological Surgery, Wakayama Medical \\ University, Wakayama, Japan
}


Conclusion Pretreatment with pitavastatin significantly reduced the frequency of periprocedural ischemic complications with CAS.

Keywords Statin therapy - Carotid artery stenting · Diffusion-weighted imaging · Pitavastatin · Ischemic complication

\section{Introduction}

Carotid artery stenting (CAS) for carotid artery stenosis has been effective not only in patients at high risk, but also those at conventional risk for carotid endarterectomy (CEA), and it is becoming an alternative to CEA [1]. However, the incidence of periprocedural stroke (particularly ischemic stroke) is higher with CAS than with CEA $[1,2]$. Factors that influence periprocedural ischemic complications in CAS include use of a protection device, stent design, technical expertise, patient age, and plaque characteristics [3-8].

In addition to the antihyperlipidemic effects, 3-hydroxy3-methylglutaryl-coenzyme (HMG-CoA) reductase inhibitors (statins) have the pleiotropic effects, including plaque stabilization, improvement of endothelial function, reduction in vascular inflammation, reduction of platelet aggregation, and thrombus deposition [9-11]. Statins can reduce periprocedural complications when administered before coronary intervention [12]. However, it has not been sufficiently investigated whether statins reduce periprocedural complications with CAS. We therefore investigated whether pre-CAS pitavastatin therapy could reduce the frequency of periprocedural ischemic complications with CAS.

\section{Methods}

\section{Study Population and Design}

The EPOCH-CAS (Effect of Pitavastatin On preventing is CHemic complications with CAS) study was a prospective study conducted at 11 medical centers in Japan. The study was approved by each hospital research ethics committee. All patients provided written informed consent before participation in this study.

The EPOCH-CAS study included patients who had $\geq 50 \%$ symptomatic or $\geq 80 \%$ asymptomatic carotid artery stenosis at a high risk for CEA and with no history of statin treatment (Table 1).

Patients were divided into two groups on the basis of serum low-density lipoprotein cholesterol (LDL-C) levels
Table 1 Inclusion criteria, exclusion criteria, and criteria for CEA high risk

Inclusion criteria

(1) Patient is scheduled to undergo CAS more than 4 weeks after study enrollment

(2) Angiography findings of $\geq 50 \%$ symptomatic or $\geq 80 \%$ asymptomatic carotid artery stenosis according to the North American Symptomatic CEA Trial criteria

(3) High risk of CEA

Exclusion criteria

(1) History of or under lipid-lowering treatment with a statin

(2) Severe liver dysfunction or biliary obstruction

(3) Severe renal dysfunction

(4) Any active inflammatory disorder such as connective tissue disease

(5) Nonatherosclerotic carotid disease

(6) Treatment with cyclosporine

(7) MRI contraindicated for reasons such as pacemaker insertion

(8) Considered ineligible for study participation for any other reason by the investigator or subinvestigator

Criteria for CEA high risk (at least one factor required)

(1) Severe left ventricular dysfunction with congestive heart failure (New York Heart Association (NYHA) class III/IV) and/ or left ventricular ejection fraction $<30 \%$

(2) Recent myocardial infarction ( $>24 \mathrm{~h}$ and $<4$ weeks)

(3) Unstable angina

(4) Open-heart surgery within 6 weeks of study enrollment

(5) Coexisting severe coronary artery disease requiring openheart surgery

(6) Severe pulmonary disease

(7) Contralateral carotid occlusion

(8) Contralateral laryngeal nerve palsy

(9) Previous radical neck surgery or radiation therapy to the neck

(10) Recurrent stenosis after CEA

(11) High cervical internal carotid artery lesion or common carotid artery lesions below the clavicle

(12) Age $\geq 75$ years

(13) Judged to be high risk by physician for any reasons other than the above

$C E A$ carotid endarterectomy, $M R I$ magnetic resonance imaging

at the time of enrollment. Patients with LDL-C $\geq 120 \mathrm{mg} /$ $\mathrm{dl}$ were provided $4 \mathrm{mg} /$ day of pitavastatin and underwent CAS 4 weeks later (pitavastatin-treated [PS] group). Patients with LDL-C $<120 \mathrm{mg} / \mathrm{dl}$ received no statin therapy and underwent CAS 4 weeks later (non-PS group). This is because Japan Atherosclerosis Society Guidelines for Prevention of Atherosclerotic Cardiovascular Diseases 2007 recommended that LDL-C level be $<120 \mathrm{mg} / \mathrm{dl}$ for patients with carotid artery stenosis. Patients in both groups underwent CAS in a nonblinded manner. Blood samples were collected on the day before CAS in both groups. 


\section{CAS Procedure}

Dual antiplatelet therapy (aspirin [100 mg] and clopidogrel [75 mg] or cilostazol [200 mg]) was initiated at least 1 week before CAS and continued for at least for 30 days after CAS. Use of an embolic protection device (EPD) was mandatory, but the type was unrestricted. The type of stent used was also not restricted. Pre- and postdilation were performed when necessary during the procedure.

Magnetic Resonance Imaging Technique

\section{Diffusion-Weighted Imaging (DWI)}

Each center was allowed to use scanners with field strengths of 1.5 or $3 \mathrm{~T}$, as long as the same scanner and the same imaging parameters were used in both groups. The imaging protocol included the same DWI sequences $\left(b=0,1,000 \mathrm{~s} / \mathrm{mm}^{2}\right.$; section thickness $5.0 \mathrm{~mm}$; gap $1 \mathrm{~mm}$ ); apparent diffusion coefficient maps were obtained in all cases. The protocol specified magnetic resonance (MR) imaging scans to take place within 1 week before CAS and within 12-72 h after CAS. The window/level setting during evaluation was set on the basis of previously published standards for DWI [13]. Two board-certified neuroradiologists independently and blindly analyzed all scans, and they evaluated the number of lesions measuring $<10 \mathrm{~mm}$ and $\geq 10 \mathrm{~mm}$ separately, as well as the vascular distribution. DWI positivity was defined by at least one new bright lesion on DWI that had not been detected at baseline. Differences in assessment were resolved by consensus.

\section{MR Plaque Imaging}

MR imaging for plaque characterization was performed on 1.5 or $3 \mathrm{~T}$ scanners. For each patient, follow-up studies were performed with the same imaging unit as the baseline study. A 2D T1-weighted fast spin-echo sequence was performed before and after 4-week statin treatment using a black blood double inversion recovery preparation pulse and a fat-saturation pulse. The signal intensities were measured with the region of interest drawn over the carotid plaque at the most severely stenotic level. The signal intensity ratio of the plaque relative to adjacent muscle was calculated.

\section{End Points}

The primary end point of this study was the incidence of new ipsilateral ischemic lesions on DWI, and the secondary end point was the occurrence of cerebrovascular events (defined as transient ischemic attack, stroke, or death) within 30 days after CAS. Stroke was defined as a neurological deficits that persisted for more than $24 \mathrm{~h}$.

\section{Statistical Analysis}

Data were expressed as mean \pm standard deviation for continuous variables and as frequencies for categorical variables. Continuous variables were compared by the two sample $t$ test or the Wilcoxon rank sum test, and categorical variables by the $\chi^{2}$ test or Fisher's exact test. A multiple logistic regression analysis was constructed to identify independent predictors of new ipsilateral ischemic lesions on DWI. Statistical significance was defined as a two-sided $P$ value of $<0.05$. JMP version 9 (SAS Institute, Cary, NC, USA) was used for all statistical analyses.

\section{Results}

\section{Study Profile}

A total of 80 patients were enrolled (PS group, $n=44$; non-PS group, $n=36$ ) from July 2010 to September 2012 . Eight patients withdrew from the study for the following reasons: canceled CAS, 3 patients in the PS group (malignant tumor in 2 and ileus in 1); emergency CAS, 1 in the PS group and 1 in the non-PS group; and stopped pitavastatin therapy ( 3 in the PS group-creatine kinase elevation in 2 and patient preference in 1) (Fig. 1). Two patients who underwent emergency CAS because of minor ischemic stroke during the waiting period were discharged without any neurological sequela.

CAS was performed successfully in all 72 patients (PS group, $n=37$; non-PS group, $n=35$ ) meeting the inclusion criteria. Eleven patients (six in the PS group and five in the non-PS group) were excluded because they were in violation of the DWI protocol, as follows: preprocedural DWI more than 1 week before CAS, 4 in the PS group and 4 in the non-PS group; no preprocedural DWI, 1 in the PS group; different slice thickness $(7 \mathrm{~mm}), 1$ in the PS group; and preprocedural DWI more than 1 week before CAS with different slice thickness $(3 \mathrm{~mm}), 1$ in the non-PS group. A total of 61 patients (PS group, $n=31$; non-PS group, $n=30$ ) underwent the primary end-point analyses (Fig. 1).

In these 61 patients, cholesterol levels were significantly different between the two groups. At the time of study enrollment, LDL-C and total cholesterol levels were significantly higher in the PS group than in the non-PS group; in contrast, on the day before CAS, LDL-C and total cholesterol levels were significantly lower in the PS group than in the non-PS group (Table 2). The findings of MR 
Fig. 1 Study profile. $P S$ pitavastatin treated, $C A S$ carotid artery stenting, $L D L-C$ lowdensity lipoprotein cholesterol, $D W I$ diffusion-weighted imaging

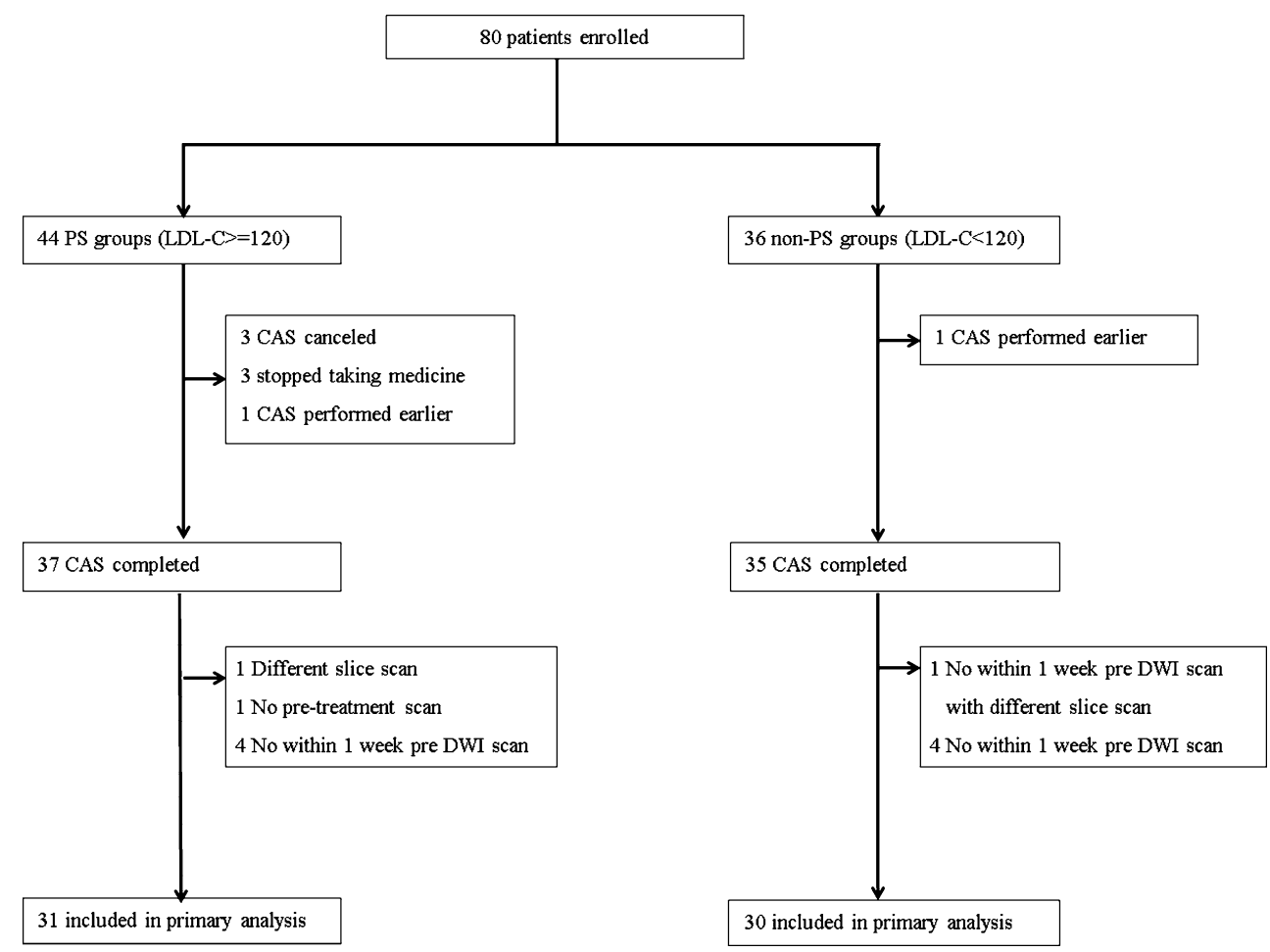

Table 2 Changes of lipids levels at the baseline and before CAS

\begin{tabular}{|c|c|c|c|c|c|c|}
\hline \multirow[t]{2}{*}{ Lipid } & \multicolumn{3}{|l|}{ Baseline } & \multicolumn{3}{|l|}{ Before CAS } \\
\hline & PS group $(n=31)$ & Non-PS group $(n=30)$ & $P$ value & PS group $(n=31)$ & Non-PS group $(n=30)$ & $P$ value \\
\hline Total cholesterol, mg/dl & $218 \pm 29$ & $175 \pm 51$ & 0.0001 & $135 \pm 23$ & $166 \pm 34$ & 0.0001 \\
\hline Triglycerides, mg/dl & $127 \pm 55$ & $133 \pm 77$ & 0.69 & $96 \pm 44$ & $133 \pm 72$ & 0.02 \\
\hline HDL cholesterol, mg/dl & $47 \pm 12$ & $48 \pm 17$ & 0.83 & $47 \pm 12$ & $48 \pm 19$ & 0.77 \\
\hline LDL cholesterol, mg/dl & $145 \pm 22$ & $93 \pm 21$ & $<0.0001$ & $69 \pm 16$ & $91 \pm 24$ & 0.0001 \\
\hline
\end{tabular}

Data are presented as mean \pm standard deviation

$C A S$ carotid artery stenting, $P S$ pitavastatin treated, $H D L$ high-density lipoprotein, $L D L$ low-density lipoprotein

Table 3 Changes of signal intensity ratio in the MR plaque imaging

\begin{tabular}{|c|c|c|c|c|c|c|c|}
\hline \multicolumn{3}{|c|}{ PS group $(n=31)$} & \multicolumn{3}{|c|}{ Non-PS group $(n=30)$} & \multirow{2}{*}{$\begin{array}{l}P \text { value (PS vs. } \\
\text { non-PS) at baseline }\end{array}$} & \multirow{2}{*}{$\begin{array}{l}P \text { value } \\
\text { (PS vs. non-PS) } \\
\text { at pre-CAS }\end{array}$} \\
\hline Baseline & Before CAS & $\begin{array}{l}P \text { value } \\
\text { (vs. baseline) }\end{array}$ & Baseline & Before CAS & $\begin{array}{l}P \text { value } \\
\text { (vs. baseline) }\end{array}$ & & \\
\hline $1.327 \pm 0.268$ & $1.368 \pm 0.344$ & 0.6089 & $1.368 \pm 0.344$ & $1.333 \pm 0.297$ & 0.2070 & 0.9854 & 0.6202 \\
\hline
\end{tabular}

Data are presented as mean \pm standard deviation

$M R$ magnetic resonance, $P S$ pitavastatin treated, $C A S$ carotid artery stenting

plaque imaging were similar between both groups in each analysis at both baseline and before CAS (Table 3). There were also no significant differences in the signal intensity ratio between the pre- and poststatin treatment times in the PS group. Other baseline clinical characteristics and procedural data did not differ significantly between the groups (Table 4).

\section{New Ischemic Lesions on DWI}

New ipsilateral ischemic lesions were detected in 24 of the 61 patients $(39.3 \%)$; the DWI-positive rate was significantly lower in the PS group ( $25.8 \%, 8$ of 31 patients) than in the non-PS group $(53.3 \%, 16$ of 30 patients; $P=0.028$ ) (Table 5). 
Table 4 Demographics and baseline characteristics

\begin{tabular}{|c|c|c|c|}
\hline Characteristic & $\begin{array}{l}\text { PS group } \\
(n=31)\end{array}$ & $\begin{array}{l}\text { Non-PS } \\
\text { group } \\
(n=30)\end{array}$ & $P$ value \\
\hline Male, $n(\%)$ & $26(83.9)$ & $29(96.7)$ & 0.09 \\
\hline Age, year & $72 \pm 9$ & $74 \pm 8$ & 0.31 \\
\hline Degree of stenosis $(\%)$ & $79 \pm 11$ & $79 \pm 12$ & 0.97 \\
\hline \multicolumn{4}{|l|}{ History, $n(\%)$} \\
\hline Symptomatic stenosis & $14(45.2)$ & $14(46.7)$ & 0.91 \\
\hline Ischemic heart disease & $5(16.1)$ & $4(13.3)$ & 0.76 \\
\hline Hypertension & $21(67.7)$ & $19(63.3)$ & 0.72 \\
\hline Diabetes mellitus type 2 & $12(38.7)$ & $6(20.0)$ & 0.11 \\
\hline Hyperlipidemia & $31(100.0)$ & $0(0.0)$ & $<0.0001$ \\
\hline Current or past smoker & $18(58.1)$ & $16(53.3)$ & 0.71 \\
\hline \multicolumn{4}{|l|}{ High risk for CEA } \\
\hline $\begin{array}{l}\text { CHF and/or known } \\
\text { severe LVEF }\end{array}$ & $0(0.0)$ & $0(0.0)$ & - \\
\hline Recent MI & $0(0.0)$ & $0(0.0)$ & - \\
\hline Unstable angina & $2(6.5)$ & $2(6.7)$ & 0.97 \\
\hline $\begin{array}{l}\text { Open heart surgery } \\
\text { needed within } 6 \text { wk }\end{array}$ & $0(0.0)$ & $0(0.0)$ & - \\
\hline $\begin{array}{l}\text { Coexisting severe } \\
\text { coronary artery } \\
\text { disease }\end{array}$ & $1(3.2)$ & $1(3.3)$ & 0.98 \\
\hline $\begin{array}{l}\text { Severe pulmonary } \\
\text { disease }\end{array}$ & $0(0.0)$ & $0(0.0)$ & - \\
\hline $\begin{array}{l}\text { Contralateral carotid } \\
\text { occlusion }\end{array}$ & $1(3.2)$ & $1(3.3)$ & 0.98 \\
\hline $\begin{array}{l}\text { Contralateral laryngeal } \\
\text { nerve palsy }\end{array}$ & $0(0.0)$ & $0(0.0)$ & - \\
\hline $\begin{array}{l}\text { Previous radical neck } \\
\text { surgery or radiation } \\
\text { therapy to the neck }\end{array}$ & $0(0.0)$ & $2(6.7)$ & 0.14 \\
\hline $\begin{array}{l}\text { Recurrent stenosis after } \\
\text { CEA }\end{array}$ & $0(0.0)$ & $0(0.0)$ & - \\
\hline $\begin{array}{l}\text { High cervical ICA } \\
\text { lesions or CCA lesions } \\
\text { below the clavicle }\end{array}$ & $10(32.3)$ & $10(33.3)$ & 0.93 \\
\hline Age $>75$ years & $7(22.6)$ & $11(36.7)$ & 0.23 \\
\hline Physician judgment & $9(29.0)$ & $7(23.3)$ & 0.61 \\
\hline \multicolumn{4}{|l|}{ EPD } \\
\hline \multicolumn{4}{|l|}{ Distal balloon protection } \\
\hline PercuSurge (PerS) & $0(0.0)$ & $1(3.3)$ & 0.31 \\
\hline \multicolumn{4}{|l|}{ Distal filter protection } \\
\hline Angioguard RX & $1(3.2)$ & $0(0.0)$ & 0.32 \\
\hline FilterWire EZ (FW) & $23(74.2)$ & $26(86.7)$ & 0.22 \\
\hline Spider FX & $3(9.7)$ & $0(0.0)$ & 0.08 \\
\hline \multicolumn{4}{|l|}{$\begin{array}{l}\text { Multiple protection } \\
\text { (proximal protection } \\
\text { (PP) }+ \text { distal } \\
\text { protection) }\end{array}$} \\
\hline $\mathrm{PP}+\mathrm{FW}$ & $2(6.5)$ & $0(0.0)$ & 0.16 \\
\hline $\mathrm{PP}+\mathrm{PerS}$ & $1(3.2)$ & $1(3.3)$ & 0.98 \\
\hline $\mathrm{PP}+\mathrm{FW}+\mathrm{PerS}$ & $1(3.2)$ & $2(6.7)$ & 0.53 \\
\hline
\end{tabular}

Table 4 continued

\begin{tabular}{lccc}
\hline Characteristic & $\begin{array}{l}\text { PS group } \\
(n=31)\end{array}$ & $\begin{array}{l}\text { Non-PS } \\
\text { group } \\
(n=30)\end{array}$ & $P$ value \\
\hline $\begin{array}{l}\text { Stent } \\
\text { Open cell }\end{array}$ & & \\
$\quad \begin{array}{l}\text { Precise } \\
\text { Protégé }\end{array}$ & $10(32.3)$ & $14(46.7)$ & 0.25 \\
Closed cell & $1(3.2)$ & $0(0.0)$ & 0.32 \\
$\quad$ & & \\
Carotid Wallstent & $20(64.5)$ & $16(53.3)$ & 0.37 \\
\hline $\begin{array}{l}\text { Data are presented as mean } \pm \text { standard deviation or } n(\%) \\
P S \text { pitavastatin treated, } C E A \text { carotid endarterectomy, } C H F \text { chronic }\end{array}$ \\
$\begin{array}{l}\text { heart failure, } L V E F \text { left ventricular ejection fraction, } M I \text { myocardial } \\
\text { infarction, } I C A \text { internal carotid artery, } C C A \text { common carotid artery }\end{array}$
\end{tabular}

Table 5 New DWI-positive lesions on posttreatment scans

\begin{tabular}{llll}
\hline Lesion & $\begin{array}{l}\text { PS group } \\
(n=31)\end{array}$ & $\begin{array}{l}\text { Non-PS } \\
\text { group } \\
(n=30)\end{array}$ & $P$ value \\
\hline $\begin{array}{l}\text { Ipsilateral new lesion (at least } \\
\text { one new lesion) }\end{array}$ & $8(25.8 \%)$ & $16(53.3 \%)$ & 0.028 \\
$\begin{array}{l}\text { Ipsilateral single new lesions } \\
\begin{array}{l}\text { Ipsilateral multiple new } \\
\text { lesions }\end{array}\end{array}$ & $5(16.1 \%)$ & $3(10.0 \%)$ & - \\
$\begin{array}{l}\text { Ipsilateral and nonipsilateral } \\
\text { (contralateral or posterior } \\
\text { circulation) new lesion }\end{array}$ & $8(25.8 \%)$ & $13(43.3 \%)$ & - \\
\hline
\end{tabular}

$D W I$ diffusion-weighted imaging, $P S$ pitavastatin treated

In three patients (two in the PS group and one in the non-PS group), new ischemic lesions were also observed on the contralateral side, and three patients from the nonPS group showed ischemic lesions in the posterior circulation. In five patients, except for one patient in the non-PS group, ischemic lesions were also observed on the ipsilateral side. When we included the contralateral side and the posterior circulation lesion, the DWI positivity rate was also significantly lower in the PS group than in the non-PS group $(P=0.014)$ (Table 5).

The mean number of ischemic lesions was 4.67 (range 1-42): the median number of lesions was 1 (range 1-8) in the PS group and 2 (range 1-42) in the non-PS group. The multiple lesion rates on the ipsilateral side were $9.7 \%$ (3 of 31 patients) in the PS group and $43.3 \%$ (13 of 30 patients) in the non-PS group. One patient with transient ischemic attack and 2 patients with minor stroke in the non-PS group had multiple lesions (3, 12, and 42 lesions, respectively). Other DWI-positive lesions were all asymptomatic.

As to lesion size, 5 lesions were $10-20 \mathrm{~mm}$ in diameter, and all other lesions were under $10 \mathrm{~mm}$. One minor stroke patient had 4 lesions of $\geq 10 \mathrm{~mm}$. Another lesion of $\geq 10 \mathrm{~mm}$ was asymptomatic. 
Table 6 Cerebrovascular events within 30 days

\begin{tabular}{llll}
\hline Event & $\begin{array}{l}\text { PS group } \\
(n=31)\end{array}$ & $\begin{array}{l}\text { Non-PS group } \\
(n=30)\end{array}$ & $P$ value \\
\hline Cerebrovascular events & 0 & $3(10.0 \%)$ & 0.071 \\
Stroke & 0 & $2(6.7 \%)$ & 0.144 \\
Transient ischemic attack & 0 & $1(3.3 \%)$ & 0.305 \\
Death & 0 & 0 & - \\
\hline
\end{tabular}

$P S$ pitavastatin treated

\section{Cerebrovascular Events}

Of 61 patients, three patients ( $4.9 \%$ ), all of whom belonged to the non-PS group, had cerebrovascular events; this included transient ischemic attack in one patient $(1.6 \%)$ and minor ischemic stroke in two patients ( $3.3 \%)$. In the both patients with stroke, the symptoms completely disappeared within 30 days. No deaths occurred in either group. As a result, cerebrovascular events tended to occur less frequently in the PS group than in the non-PS group $(P=0.071)$ (Table 6).

\section{Independent Factors Related to New Ischemic Lesions on DWI}

To determine whether the pitavastatin treatment decreased the post-CAS ischemic complications, multivariate analyses were performed using the post-CAS development of new ipsilateral DWI-positive lesion as the dependent variable. Independent variables were PS group, degree of stenosis, symptomatic stenosis, pre-CAS LDL-C levels, pre-CAS signal intensity ratio on MR plaque imaging, type of EPDs, and use of closed cell stents, all of which could potentially affect the post-CAS occurrence of ischemic complications. Multivariate analyses revealed that the PS group $(\beta=0.74,95 \%$ confidence interval $0.070-1.48$, $P=0.042)$ and degree of carotid stenosis $(\beta=0.095$, $95 \%$ confidence interval $0.030-0.18, P=0.023$ ) were independently associated with less frequent occurrence of post-CAS ipsilateral ischemic lesions (Table 7).

\section{Discussion}

In the field of percutaneous coronary intervention, statin administration before intervention has already been reported to decrease periprocedural complications. A randomized study in elective coronary intervention patients indicated that the occurrence of periprocedural complications was significantly lower in the atorvastatin therapy group compared to the placebo group [12]. The mechanisms underlying the beneficial effects of atorvastatin are not completely clear. Such results are presumably the result of the pleiotropic effects of statins.
Table 7 Factors affecting the incidence of new ischemic lesions on DWI (multivariate analysis)

\begin{tabular}{lcll}
\hline Factor & $\beta$ & $\begin{array}{l}\text { 95 \% } \\
\text { confidence } \\
\text { interval }\end{array}$ & $P$ value \\
\hline PS group & 0.74 & 0.070 to 1.48 & 0.042 \\
Degree of stenosis at baseline & 0.095 & 0.030 to 0.18 & 0.023 \\
Symptomatic stenosis & -0.2359 & -0.95 to 0.48 & 0.51 \\
LDL-C before CAS & 0.0012 & -0.027 to 0.030 & 0.93 \\
Signal intensity ratio in MR & 0.66 & -1.49 to 2.97 & 0.55 \\
$\quad$ plaque imaging before CAS & & -0.29 to 1.57 & 0.20 \\
EPD (distal protection) & 0.59 & -0.30 to 1.08 & 0.30 \\
Stent (closed cell) & 0.37 & &
\end{tabular}

$D W I$ diffusion-weighted imaging, $P S$ pitavastatin treated, $L D L-C$ lowdensity lipoprotein cholesterol, $C A S$ carotid artery stenting, EPD embolic protection device

To our knowledge, only two retrospective studies have reported the possible benefits of statins on CAS. Groschel et al. [14] reported that the periprocedural cardiovascular events rate in 180 CAS cases was significantly lower than in various prestatin treatment groups $(4 \%)$ than in the nonprestatin therapy group $(15 \%)$.

Verzini et al. [15] provided a retrospective analysis of 1,083 consecutive CAS cases. Statin use was associated with a reduction in risk of perioperative stroke and death (odds ratio $0.327 ; 95 \%$ confidence interval $0.13-0.80$, $P=0.016$ ). Our study showed that the cerebrovascular event rate in the PS group was not significantly lower than in the non-PS group. Nevertheless, a significant difference was evident on DWI, so if the number of patients were increased, a significant difference even for the cerebrovascular event might be identified.

Kastrup et al. [16] reported that the number of new DWI lesions was significantly higher in patients who developed a stroke than in patients without neurological deficit. Major stroke patients had multiple lesions larger than $20 \mathrm{~mm}$ in diameter, whereas all patients with minor stroke had multiple new lesions smaller than $10 \mathrm{~mm}$ in diameter. In our study, two minor stroke patients also had multiple lesions (12 and 42); the size of lesions was also smaller than $10 \mathrm{~mm}$ in diameter except for 4 lesions of $10-20 \mathrm{~mm}$.

Several investigators, using DWI to detect clinically silent embolic lesions after protected CAS, have reported new DWI lesions at frequencies of $6.7-73 \%$ [8, 17-21]. A substudy of the International Carotid Stenting Study (ICSS) reported that new DWI lesions occurred in 62 of 124 patients $(50 \%)$ in the stenting group [18]. In a DWIpositive CAS group (62 patients), single lesions were seen in $18(15 \%)$, multiple lesions in $44(35 \%)$, and stroke in 8 (6\%). New DWI lesions were present in $73 \%$ in an EPD group and $34 \%$ in a group without EPD. In this study, EPD 
was used in all cases, and new DWI lesions occurred in $25.8 \%$ (8 of 31) in the PS group, which was much lower compared with $56.7 \%$ (17 of 30) in the non-PS group in this study and $73 \%$ in EPD group in the ICSS study. Moreover, both the ICSS study and the non-PS group in this study showed that CAS-related embolisms much more frequently occurred as multiple lesions, whereas the frequency of multiple lesions was much lower than that of single lesion in the PS group. These findings suggest that preprocedural statin therapy is effective for reduction of both number and occurrence rate of ischemic lesions.

In our study, there was a significant difference in LDL-C and total cholesterol levels between the two groups. However, several authors reported that dyslipidemia or hyperlipidemia did not affect the post-CAS DWI-positive lesion occurrence $[19,21]$. Our study also revealed that an independent factor for post-CAS new DWI-positive lesions was pitavastatin treatment, but not LDL-C level. Taken together, these findings suggest that the statin therapy reduced post-CAS DWI-positive lesions with the pleiotropic effects other than improving cholesterol profiles.

In our study, we chose pitavastatin out of many statins because only pitavastatin can reportedly stabilize the carotid plaque within 1 month after administering statins. Nakamura et al. [10] reported that plaque stabilization of unstable carotid plaque significantly occurred by pitavastatin therapy within 1 month on ultrasonography. Yoshimura et al. [8] reported that unstable plaque on time-of-flight MR angiography was associated with more ipsilateral ischemic lesions on DWI after CAS. One possible explanation for the lower DWIpositive rate in the PS group in this study is plaque stabilization as a consequence of the pitavastatin administration. However, in our study, there was no significant difference in MR plaque imaging findings between pre- and poststatin treatment in the PS groups. This result suggests that the lower DWI-positive rate in the PS group may not be due to plaque stabilization but rather to other pleiotropic effects. Otherwise, MR plaque imaging in the current study may not be suitable for detecting the short-term changes in plaque stability. We have evaluated plaque stability using T1-weighted MR plaque imaging because several reports described that high-signal plaques on T1-weighted images predict silent ischemic lesions after CAS [22, 23]. However, ultrasound may be much more sensitive than MR plaque imaging regarding plaque stability. Therefore, it would be expected that ultrasound is also added in the protocol in a future study.

New ischemic lesions, even without corresponding focal deficit, might lead to long-term clinical consequences, including cognitive decline and dementia [24]. Thus, reducing ischemic lesions as much as possible is essential in CAS. For this purpose, statins are promising.

There are some limitations in this study, as follows. This study had a nonrandomized design with a relatively small sample size; there were significant differences in LDL-C levels between the groups; different stents and EPDs were used; DWI was performed by different MR imaging machines; pre-CAS DWI scan was performed within 1 week before CAS; and efficacy of antiplatelet agents was not evaluated. Despite these limitations, this is to our knowledge the first prospective study at multiple centers to demonstrate that pretreatment with pitavastatin decreases the incidence of ischemic complication after CAS. Future randomized control trials are now warranted.

\section{Conclusion}

Pretreatment with pitavastatin significantly reduced periprocedural ischemic complications with CAS.

Acknowledgments We acknowledge Dr. Hiro Kiyosue (Department of Radiology, Oita University Faculty of Medicine).

Conflict of interest The authors declare that they have no conflict of interest.

\section{Appendix}

The investigators and institutions participating in the EPOCHCAS study were as follows: Ishinkai Yao General Hospital, Yao, Osaka (Katsutoshi Takayama, MD, Kaoru Myouchin, MD, Ryota Kimura, MD, Shinichiro Kurokawa, MD); Osaka Medical College, Takatsuki, Osaka (Naokado Ikeda, MD, Terumasa Kuroiwa, MD, Toshihiko Kuroiwa, MD); Kyoto First Red Cross Hospital, Kyoto (Keisuke Imai, MD, Masashi Hamanaka, MD); Kokura Memorial Hospital, Fukuoka (Masanori Gomi, MD, Ichiro Nakahara, MD); Shiroyama Hospital, Habikino, Osaka (Kosuke Miyake, MD, Kenichi Murao, MD); Suzuka General Hospital, Suzuka, Mie (Ryuta Yasuda, MD, Kenji Kawaguchi, MD); Suzuka Kaisei Hospital, Suzuka, Mie (Kazuhide Hamada, MD, Kenji Kanamaru, MD); Nara Medical University, Kashihara, Nara (Takeshi Wada, MD, Ichiro Nakagawa, MD, Masahiko Sakamoto, MD, Toshiaki Taoka, MD, Hiroyuki Nakase, MD, Kimihiko Kichikawa, MD); Mie University Graduate School of Medicine, Tsu, Mie (Hiroshi Tanemura, MD, Naoki Toma, MD, Masayuki Maeda, MD, Hidenori Suzuki, MD, Waro Taki, MD); Yagi Neurosurgical Hospital, Osaka (Hiroyuki Ohnishi, MD, Hirokatsu Taniguchi, MD); Wakayama Medical University, Wakayama (Arisa Umesaki, MD, Osamu Masuo, MD, Naoyuki Nakao, MD).

\section{References}

1. Brott TG, Hobson RW 2nd, Howard G et al (2010) Stenting versus endarterectomy for treatment of carotid-artery stenosis. N Engl J Med 363:11-23 
2. International Carotid Stenting Study Investigators (2010) Carotid artery stenting compared with endarterectomy in patients with symptomatic carotid stenosis (International Carotid Stenting Study): an interim analysis of a randomised controlled trial. Lancet 375(9719):985-997

3. Jansen O, Fiehler J, Hartmann M, Bruckmann H (2009) Protection or nonprotection in carotid stent angioplasty: the influence of interventional techniques on outcome data from the SPACE Trial. Stroke 40:841-846

4. Lal BK, Brott TG (2009) The Carotid Revascularization Endarterectomy vs Stenting Trial completes randomization: lessons learned and anticipated results. J Vasc Surg 50:1224-1231

5. Hobson RW 2nd, Howard VJ, Roubin GS et al (2004) Carotid artery stenting is associated with increased complications in octogenarians: 30-day stroke and death rates in the CREST leadin phase. J Vasc Surg 40:1106-1111

6. Bosiers M, de Donato G, Deloose K et al (2007) Does free cell area influence the outcome in carotid artery stenting? Eur J Vasc Endovasc Surg 33:135-141

7. Hopkins LN, Roubin GS, Chakhtoura EY et al (2010) The Carotid Revascularization Endarterectomy versus Stenting Trial: credentialing of interventionalists and final results of lead-in phase. J Stroke Cerebrovasc Dis 19:153-162

8. Yoshimura S, Yamada K, Kawasaki M et al (2011) High-intensity signal on time-of-flight magnetic resonance angiography indicates carotid plaques at high risk for cerebral embolism during stenting. Stroke 42:3132-3137

9. Davignon J (2012) Pleiotropic effects of pitavastatin. Br J Clin Pharmacol 73:518-535

10. Nakamura T, Obata JE, Kitta Y et al (2008) Rapid stabilization of vulnerable carotid plaque within 1 month of pitavastatin treatment in patients with acute coronary syndrome. J Cardiovasc Pharmacol 51:365-371

11. Watanabe K, Sugiyama S, Kugiyama K et al (2005) Stabilization of carotid atheroma assessed by quantitative ultrasound analysis in nonhypercholesterolemic patients with coronary artery disease. J Am Coll Cardiol 46:2022-2030

12. Pasceri V, Patti G, Nusca A et al (2004) Randomized trial of atorvastatin for reduction of myocardial damage during coronary intervention: results from the ARMYDA (Atorvastatin for Reduction of MYocardial Damage during Angioplasty) study. Circulation 110:674-678
13. Sasaki M, Ida M, Yamada K et al (2007) Standardizing display conditions of diffusion-weighted images using concurrent b0 images: a multi-vendor multi-institutional study. Magn Reson Med Sci 6:133-137

14. Gröschel K, Ernemann U, Schulz JB et al (2006) Statin therapy at carotid angioplasty and stent placement: effect on procedure-related stroke, myocardial infarction, and death. Radiology 240:145-151

15. Verzini F, De Rango P, Parlani G et al (2011) Effects of statins on early and late results of carotid stenting. J Vasc Surg 53:71-79

16. Kastrup A, Nagele T, Groschel K et al (2006) Incidence of new brain lesions after carotid stenting with and without cerebral protection. Stroke 37:2312-2316

17. Takayama $K$, Taoka $T$, Nakagawa $H$ et al (2011) Initial experience of carotid artery stenting using the Carotid Wallstent and FilterWire EZ in Japan. Jpn J Radiol 29:51-58

18. Bonati LH, Jongen LM, Haller S et al (2010) New ischaemic brain lesions on MRI after stenting or endarterectomy for symptomatic carotid stenosis: a substudy of the International Carotid Stenting Study (ICSS). Lancet Neurol 9:353-362

19. Stojanov D, Ilic M, Bosnjakovic P et al (2012) New ischemic brain lesions on diffusion-weighted MRI after carotid artery stenting with filter protection: frequency and relationship with plaque morphology. AJNR Am J Neuroradiol 33:708-714

20. Schnaudigel S, Groschel K, Pilgram SM, Kastrup A (2008) New brain lesions after carotid stenting versus carotid endarterectomy: a systematic review of the literature. Stroke 39:1911-1919

21. Russjan A, Goebell E, Havemeister S et al (2012) Predictors of periprocedural brain lesions associated with carotid stenting. Cerebrovasc Dis 33:30-36

22. Yamada K, Kawasaki M, Yoshimura S et al (2010) Prediction of silent ischemic lesions after carotid artery stenting using integrated backscatter ultrasound and magnetic resonance imaging. Atherosclerosis 208:161-166

23. Tanemura $H$, Maeda $M$, Ichikawa $N$ et al (2013) High-risk plaque for carotid artery stenting evaluated with 3-dimensional T1weighted gradient echo sequence. Stroke 44:105-110

24. Pendlebury ST, Rothwell PM (2009) Prevalence, incidence, and factors associated with pre-stroke and post-stroke dementia: a systematic review and meta-analysis. Lancet Neurol 8:1006-1018 